\title{
Corrigendum: The impact of free-ranging domestic cats on wildlife of the United States
}

\author{
Scott R. Loss, Tom Will \& Peter P. Marra
}

Nature Communications 4:1396 doi:10.1038/ncomms2380 (2013); Published 29 Jan 2013; Updated 12 Dec 2013

The original version of this Article contained incorrect estimates of the number of animals killed by free-ranging domestic cats, which were based on $90 \%$ confidence levels rather than the stated $95 \%$ confidence levels. Reanalysis of the original data after publication using 95\% confidence levels resulted in larger ranges for the estimated number of animals killed. For example, in the fifth sentence of the Abstract, the number of birds killed by free-ranging domestic cats required revision from 1.4-3.7 billion to $1.3-4.0$ billion and the number of mammals killed by free-ranging domestic cats required revision from 6.9-20.7 billion to 6.3-22.3 billion. The estimates have now been corrected throughout the PDF and HTML versions of the Article.

Unrelated to the changes above, four estimates of cat predation rates on wildlife from temperate zone studies in Supplementary Table S1 were based on partial year values that had not been adjusted to year-round estimates. The values have now been revised in Supplementary Table S1.

The original conclusions are not affected by any of the above changes. 